\title{
APPROCHE EXPÉRIMENTALE DE L'IMPACT DES OISEAUX PISCIVORES SUR UNE PISCICULTURE EXTENSIVE LITTORALE
}

\author{
M. GENARD (1) ${ }^{*}$, J. MASSE (2), C. RIGAUD (2)
}

(1) Groupe d'Études et de Recherches en Écologie Appliquée - Université de Bordeaux I Avenue des Facultés, 33405 TALENCE CEDEX (France).

(2) Centre National du Machinisme Agricole, du Génie Rural, des Eaux et des Forêts 50, Avenue de Verdun, BP 3, 33611 GAZINET CEDEX (France).

\section{RÉSUMÉ}

Une étude quantitative de la prédation par des oiseaux piscivores, le Héron Cendré (Ardea cinerea) et le Grand Cormoran (Phalacrocorax carbo) a été réalisée dans un bassin ( 2 ha) situé dans un vaste ensemble de réservoirs à poissons (140 ha en eau saumâtre) du littoral atlantique.

Deux enclos, l'un protégé de la prédation par des filets et des fils $(0,3 \mathrm{ha})$, l'autre accessible aux oiseaux (0,9 ha), ont été empoissonnés avec des Mulets (Chelon labrosus et Liza ramada). L'observation régulière des oiseaux a permis de constater dans les jours suivant l'empoissonnement une concentration spectaculaire mais brève des prédateurs, hérons et cormorans se partageant respectivement les zones peu profondes et profondes de l'enclos non protégé.

Le dénombrement des poissons après trois semaines d'expérimentation a montré un épuisement total du stock de poissons. Les caractéristiques essentielles de la prédation sont la rapidité d'intervention des piscivores et la faible part des prélèvements par le héron (3\% du stock initial), malgré un taux de succès élevé (nombre de prises/nombre d'attaques).

La prédation par le cormoran n'a pu être dissociée de la mortalité indirectement due aux prédateurs. Cependant, il est vraisemblable que ce prédateur soit à l'origine d'une grande part de la mortalité correspondante, évaluée entre 64 et $97 \%$ du stock initial. L'importance de cette prédation s'explique par la fréquentation et l'intensité de la pêche (nombre d'attaques par minute) plus élevées que chez le héron.

\section{EXPERIMENTAL APPROACH TO THE PREDATION BY PISCIVOROUS BIRDS IN EXTENSIVE WATER AQUACULTURE}

\section{SUMMARY}

The predation by the Grey Heron Ardea cinerea and the Cormorant Phalacrocorax carbo has been studied in a dyked area intended for extensive brackish water aquaculture on the French atlantic coast. A pond was divided in two parts, one of which $(0,9 \mathrm{ha})$ was protected with nets and threads, the other one $(0,3 \mathrm{ha})$ left without protection.

\footnotetext{
* Nouvelle adresse au $1^{\mathrm{er}}$ mars 1993 :

Station d'Agronomie, INRA, Domaine de St Paul, 84143 MONTFAVET CEDEX (France).
} 
Fish (Mugilidae) were stocked in November, during the wintering period of the birds. Three weeks later, remaining fish in both parts of the ponds were counted. During experimentation, number, presence and predation behaviour of birds were observed.

A spectacular but temporary gathering of birds occurred during the first days following the fish stocking. Cormorants and Grey Herons respectively used deeper and shallower areas of the pond. Intensity of predation (number of attacks/minute) was higher among cormorants.

Dominant features of predation exerted by these birds are :

- the rapid exhaustion of the fish population,

- the higher predation level by cormorants than by herons, although the latter obtains a very high success ratio (number of catches/number of attacks).

\section{INTRODUCTION}

La relation entre l'accroissement des populations de Hérons Cendrés et de Grands Cormorans et leurstatut récent d'espèces protégées a été mentionné dans divers pays européens, dont la France (EIFAC, 1988). Les exploitants piscicoles considèrent généralement que les oiseaux sont susceptibles d'effectuer des prélèvements importants sur leur cheptel et dénoncent cette évolution comme une cause d'augmentation des pertes d'exploitation. Cependant, ces impressions sont souvent sans fondement scientifique (MARION, 1983 ; PARKHURST et al., 1987), d'où l'intérêt des recherches visant à mettre en évidence les caractéristiques de la prédation exercée par les oiseaux piscivores, en particulier du point de vue quantitatif.

De telles recherches sont souvent basées sur l'étude des seules populations aviennes. En général, après en avoir évalué l'abondance et connaissant les besoins alimentaires moyens de chaque espèce, on estime, parfois avec un modèle de simulation, les quantités de poissons consommées (BOWMAKER, 1963 ; PINOWSKI et al., 1980 ; DOORNBOS, 1984 ; IM et HAFNER, 1985). Cette méthode ne permet pas d'estimer les dégâts réels : d'une part, elle les minimise en ne prenant pas en compte les poissons blessés non consommés ; d'autre part, elle les exagère en incluant des poissons qui auraient dû périr de mort naturelle ou de maladie.

D'autres auteurs (BARLOW et BOCK, 1984 ; UTSCHIK, 1984 a et b) estiment les taux de mortalité de populations de poissons soumises naturellement à des pressions de pêche différentes. Ils peuvent ainsi pallier, dans une certaine mesure, l'inconvénient mentionné. Cependant, dans ce type d'approche, les facteurs du milieu non contrôlés peuvent influer sur les populations étudiées, rendant difficile l'interprétation des résultats.

Dans la présente étude, pour éviter les problèmes évoqués, nous nous sommes placés, comme l'ont fait MARION et MARION (1987), dans un contexte expérimental comparatif, la référence étant obtenue en soustrayant une partie de la population de poissons à l'action des oiseaux. Nous avons ainsi pu examiner divers aspects de la prédation exercée par deux espèces d'oiseaux piscivores, le Héron Cendré (Ardea cinerea) et le Grand Cormoran (Phalacrocorax carbo) dans des bassins piscicoles: modalités spécifiques, spatiales et temporelles. L'inconvénient de cette démarche est de limiter la superficie de la structure expérimentale afin d'exercer un contrôle fiable du peuplement piscicole, mais elle permet de compléter les données obtenues par les méthodologies décrites précédemment.

\section{MATÉRIEL ET MÉTHODES}

\subsection{Zone d'étude}

Le domaine de Certes s'étend en bordure du Bassin d'Arcachon. II est constitué de bassins saumâtres ( 140 ha en eau), dont la vocation est l'élevage extensif de poissons (fig. 1). La prédation aviaire est un phénomène saisonnier dépendant de l'hivernage des Grands Cormorans et des Hérons Cendrés, présents en grand nombre dans la région du Bassin d'Arcachon d'Octobre à Mars (fig. 2). L'essai a été réalisé à la fin de l'automne pour se placer dans des conditions de prédation représentatives de celles rencontrées annuellement sur le domaine. 


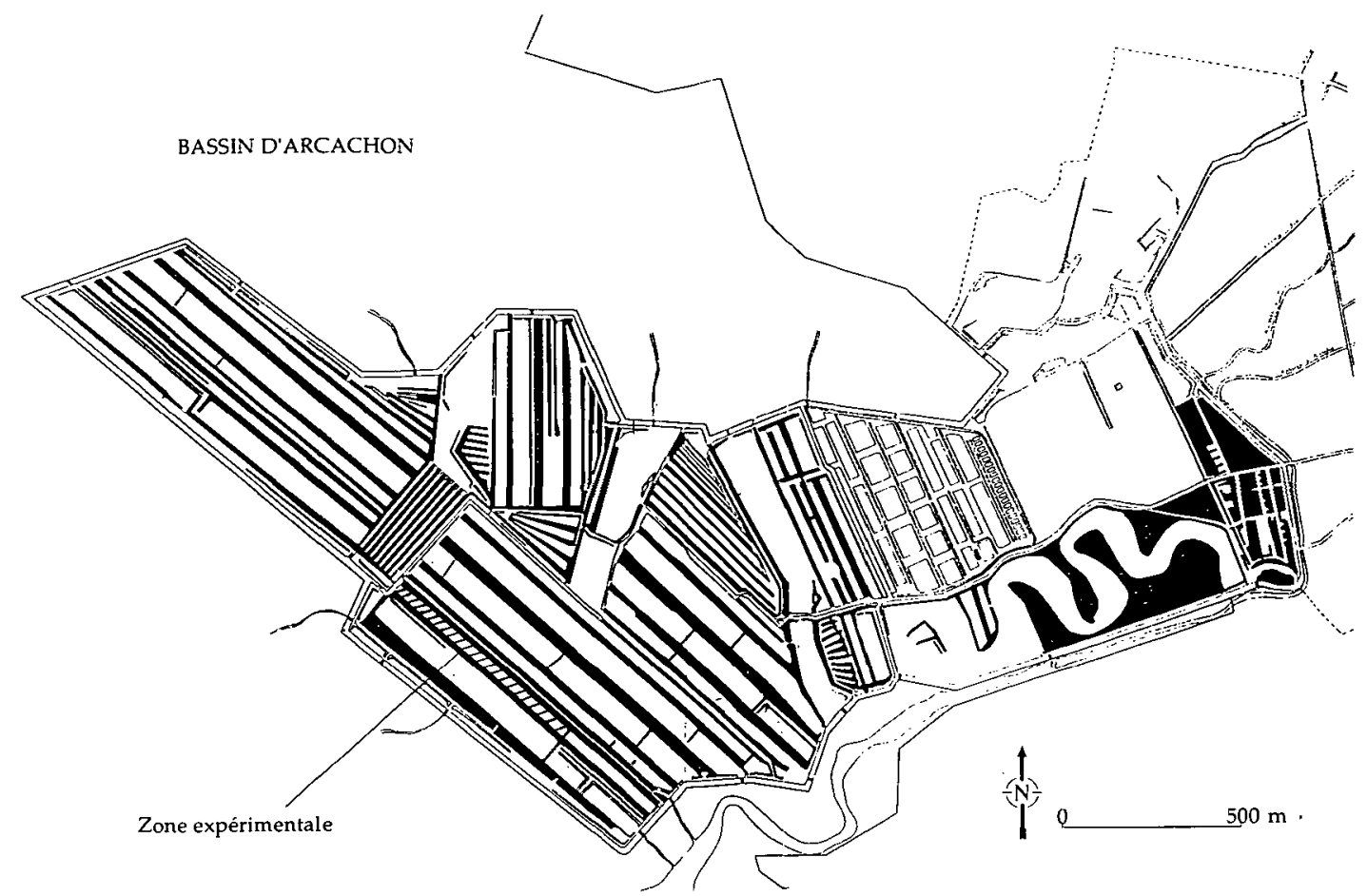

Figure 1 : Le domaine aquacole de Certes. Localisation du bassin expérimental.

Figure 1 : Aquaculture area of the "Domaine de Certes". Situation of the experimental pond.

\subsection{Dispositif expérimental}

Le choix du terrain expérimental s'est porté sur un bassin d'une superficie de 2 ha (fig. 3) de forme rectangulaire, composé d'une partie peu profonde (30 à $40 \mathrm{~cm}$ d'eau) large de $24 \mathrm{~m}$ et d'un fossé submergé ( $150 \mathrm{~cm}$ d'eau) large de $6 \mathrm{~m}$. Cette structure est comparable à celle de la plupart des réservoirs du domaine qui présentent ce profil caractéristique à double profondeur et des superficies unitaires variant de 0,5 à 5,5 ha.

Dans ce bassin, deux enclos ( 3000 et $9000 \mathrm{~m} 2$ ) sont individualisés par des séparations en grillage (maille $5 \mathrm{~mm}$ ). L'enclos de $3000 \mathrm{~m} 2$ est totalement protégé contre la prédation aviaire par des nappes de filet posées en alternance avec des fils tendus à $30 \mathrm{~cm}$ d'intervalle à une hauteur de 1,2 m au-dessus du sol. L'autre enclos n'est pas protégé. Un observatoire permet la surveillance de l'ensemble de la zone expérimentale et des proches environs.

La zone a été préparée par un assec préalable de deux mois, suivi d'une mise en eau avec filtration sur une grille de $5 \mathrm{~mm}$. Elle a été peuplée les 23 et 24 Novembre 1987 avec des poissons prélevés dans les réservoirs voisins. Les Mulets (Chelon labrosus et Liza ramada) ont été choisis, car ils constituent avec l'anguille la majeure partie des peuplements exploités du domaine (LABOURG, 1978 ; ANONYME, 1988). Le peuplement de chaque enclos est fait à partir de deux lots de poissons (tab. I et II) de tailles moyennes différentes pour juger d'une éventuelle disparité de prédation. Les poissons de chaque lot sont mélangés et répartis de façon à obtenir des densités de peuplement égales dans les deux enclos (tab. I) en respectant la distribution des tailles au pas de $1 \mathrm{~cm}$. Ces densités de peuplement voisines de 1 mulet $/ 10 \mathrm{~m} 2$ sont similaires à celles rencontrées dans les bassins du domaine. Les poissons sont introduits dans les enclos le soir pour leur permettre de se disséminer pendant la nuit. 

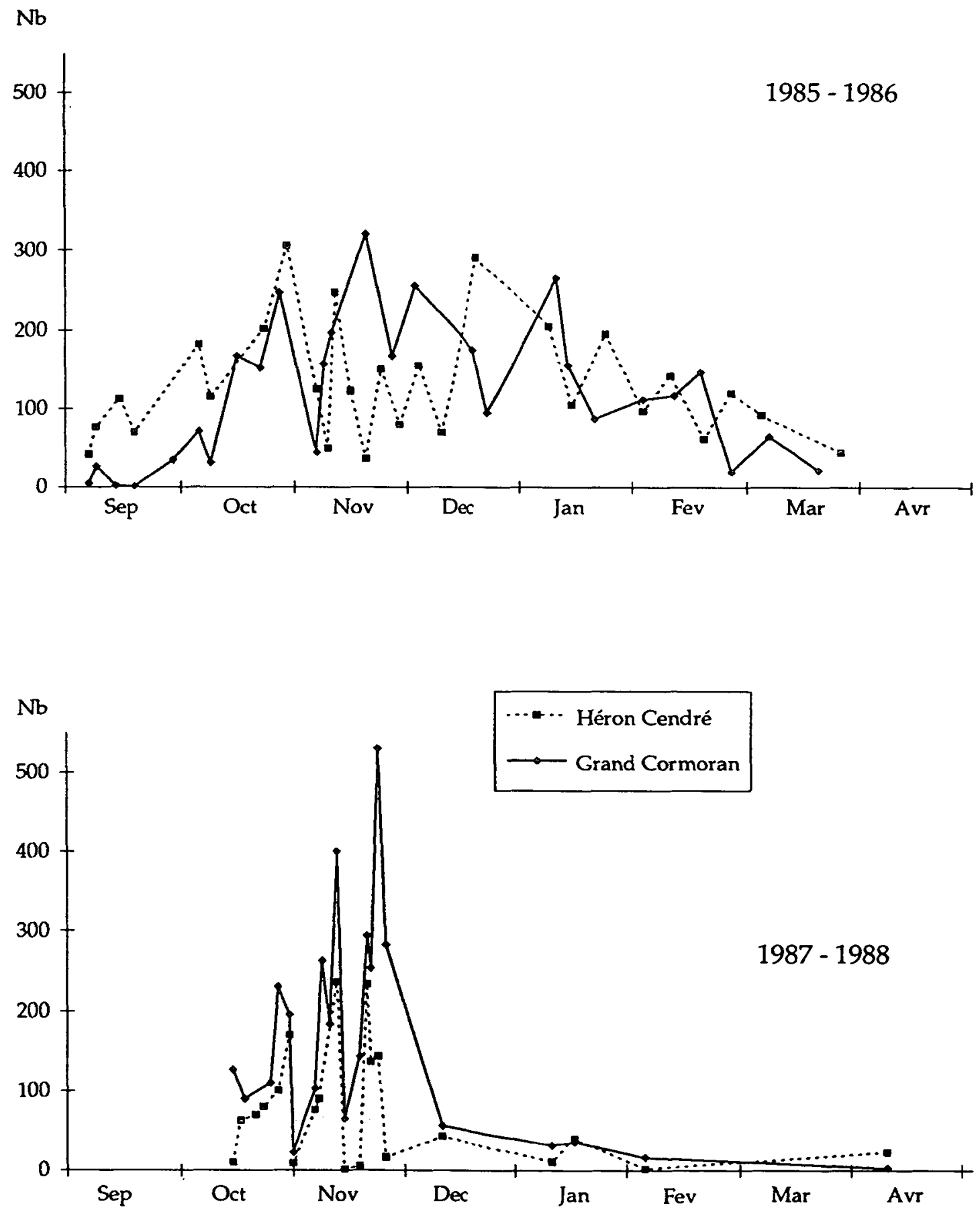

Figure 2 : Présence du Héron Cendré et du Grand Cormoran sur le domaine de Certes (comptage des oiseaux en prospection itinérante, source : CROAP).

Figure 2 : Presence of the Grey Heron and Cormorant in the Domaine de Certes (birds counting : CROAP). 


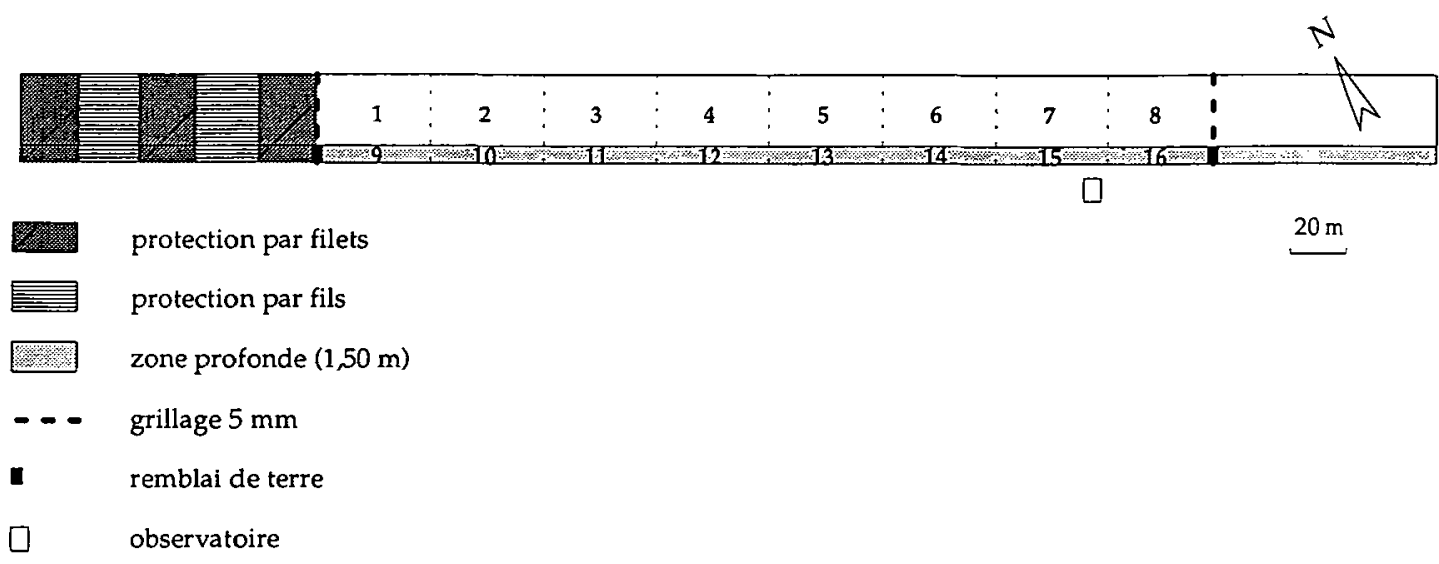

Figure 3 : Le bassin expérimental : découpage en 16 rectangles de l'enclos non protégé pour la localisation des oiseaux.

Figure 3 : Experimental pond : the unprotected enclosure is divided into 16 rectangles for birds locating.

Tableau I : Caractéristiques du peuplement en poissons de la zone expérimentale.

Table I : Characteristics of fish stocking in the experimental area.

\begin{tabular}{llcccc}
\hline & & Lot 1 & Lot 2 & Total & Charge/ha \\
\hline Enclos protégé & Nombre & 74 & 313 & 387 & 1290 \\
\cline { 2 - 6 } & Poids $(\mathrm{kg})$ & 12,2 & 14,4 & 26,6 & 88,7 \\
\hline \multirow{2}{*}{ Enclos non protégé } & Nombre & 193 & 913 & 1106 & 1229 \\
\cline { 2 - 6 } & Poids $(\mathrm{kg})$ & 31,8 & 42 & 73,8 & 82 \\
\hline
\end{tabular}

Tableau II : Caractéristiques biométriques des poissons (intervalles de confiance à $5 \%$ ). Table II : Biometric characteristics of the fishes.

\begin{tabular}{lcc}
\hline & Lot 1 & Lot 2 \\
\hline Poids moyen $(\mathrm{g})$ & $165( \pm 18)$ & $46( \pm 4)$ \\
\hline Taille minimale $(\mathrm{cm})$ & 20 & 12 \\
\hline Taille maximale $(\mathrm{cm})$ & 29 & 20 \\
\hline
\end{tabular}


L'efficacité de la cloison séparant les deux enclos a été confirmée sur la base du marquage (ablation d'une partie de la dorsale) de tous les poissons placés dans l'enclos protégé : en fin d'expérience, tous les individus survivants dans cette partie étaient marqués. Pour contrôler la mortalité induite par les marquages et les manipulations, des poissons des deux lots (62 du lot 1 et 180 du lot 2) marqués et non marqués ont été placés dans un bassin cimenté $(50 \mathrm{~m} 2)$ protégé de la prédation aviaire. La mortalité, répartie sur les 14 premiers jours, est plus grande dans le lot 2 que dans le lot 1 (respectivement $35 \%$ et $12 \%$ ). Cette différence s'explique par des conditions de pêche plus difficiles pour le lot 2 (piégeage de nuit dans une écluse). Le marquage n'a pas d'effet sur la mortalité dans le lot 1 et accentue la mortalité dans le lot $2(47 \%)$. Un facteur de correction calculé à partir de ces données a été appliqué aux résultats de l'expérimentation.

\subsection{Recueil et traitement des données}

\subsubsection{Les poissons}

Les populations de poissons ont été contrôlées le 17 Décembre par une pêche au verveux hâlé dans le creusement après abaissement du niveau d'eau. La population a été estimée selon la méthode de Carle et Strub (GERDEAUX, 1987) après trois applications successives du même effort de pêche dans chacun des deux enclos.

\subsubsection{Les oiseaux}

Le suivi des oiseaux depuis l'affût a été réalisé par journée du lever au coucher du soleil. Trois journées d'observation ont été effectuées 10 jours avant l'empoissonnement, puis huit journées dans la période allant du lendemain de l'empoissonnement jusqu'à la vidange du bassin trois semaines plus tard.

Pour chaque oiseau se posant sur la zone, il a été noté :

- l'heure d'arrivée et l'heure d'envol, rectangles,

- la situation repérée sur un quadrillage (fig. 3) découpant l'enclos non protégé en 16

- la durée de la pêche (période de nage active et de plongée pour le cormoran, période de marche et d'affûts alternés entrecoupés de tentatives de pêche pour le héron) et le nombre d'attaques (comptage direct pour le héron, nombre et durée des plongées pour le cormoran),

- le nombre de captures pour le héron (car le cormoran consomme couramment les proies sous l'eau hors de la vue de l'observateur).

La répartition temporelle de la fréquentation dans une journée donnée a été mesurée en comptabilisant le nombre de minute-oiseau enregistré sur le site (une minute-oiseau traduit la présence d'un oiseau pendant une minute). Nous avons estimé pour chaque espèce la fréquentation durant la période d'étude en recherchant, grâce à un test du c2, une fonction mathématique représentant l'occupation en fonction du temps et conforme à la distribution observée.

La répartition spatiale de chaque espèce est représentée par le cumul des observations dans chaque rectangle de repérage. Le caractère poissonnien de la répartition a été testé (test de Kologorov-Smirnov de conformité de la variable "nombre d'individus/rectangle" à une loi de Poisson de moyenne "nombre total d'observations/nombre de rectangles"). Le caractère uniforme de la répartition a également été testé (test $\mathrm{G}$ ou de $\chi 2$ de conformité de la variable aléatoire "numéro du rectangle" à une loi uniforme). Si l'hypothèse d'uniformité était rejetée, un test de contraste au risque de $5 \%$ était réalisé (sous la condition que l'hypothèse de conformité à la loi de Poisson soit aussi rejetée) pour définir les rectangles recherchés, évités ou neutres (SCHERRER, 1984).

L'intensité de la pêche (nombre total d'attaques durant la période d'étude) a été estimé pour chaque espèce, à partir d'une série discontinue de données aux dates d'observation, selon la procédure utilisée pour décrire la répartition temporelle de la fréquentation (cf. ante).

\subsubsection{Mortalité due aux prédateurs}

La perte totale induite par la prédation est égale au nombre initial de poissons placés dans l'enclos non protégé diminué du nombre de survivants et de la mortalité naturelle hors 
prédation, estimée à partir des données de l'enclos protégé. La prédation minimale est obtenue en supposant que la mortalité naturelle a lieu avant l'intervention des prédateurs.

La mortalité attribuée au héron a pu être évaluée à partir du nombre de captures relevées par comptage direct aux diverses dates d'observation. La procédure suivie est celle utilisée pour estimer la répartition temporelle et l'intensité de la pêche. N'ayant pu obtenir une telle série pour estimer les captures par le cormoran, nous avons seulement pu calculer la différence entre la mortalité totale et la mortalité attribuée au héron, sans pouvoir distinguer les pertes par prélèvement direct du cormoran et celles par stress ou blessures provoquées par l'ensemble des prédateurs (MOERBEEK et al., 1987).

\section{RÉSULTATS}

\subsection{Répartition temporelle}

Lors des 30 heures d'observation effectuées avant l'empoissonnement, 6 minutes-cormoran et 10 minutes-héron ont été enregistrées. Le nombre maximal d'oiseaux présents à un instant donné a été 2 pour les deux espèces.

Le lendemain de l'empoissonnement, 406 minutes-cormoran et 223 minutes-héron ont été enregistrées. On a pu observer, par moments, des groupes comptant jusqu'à 13 hérons ou 10 cormorans (le même jour, 150 hérons et 550 cormorans ont été recensés sur le domaine). Cette concentration d'oiseaux sur le bassin expérimental a ensuite diminué rapidement pour devenir très faible dès le 5 ème jour suivant l'introduction des poissons (fig. 4). Pour les deux espèces, aucune différence significative n'a été notée dans la fréquentation selon les heures de la journée. Pour le cormoran, cette observation diffère des résultats enregistrés dans le marais des Olonnes (BUILLES et al., 1986)

Nombre de minutes-oiseaux

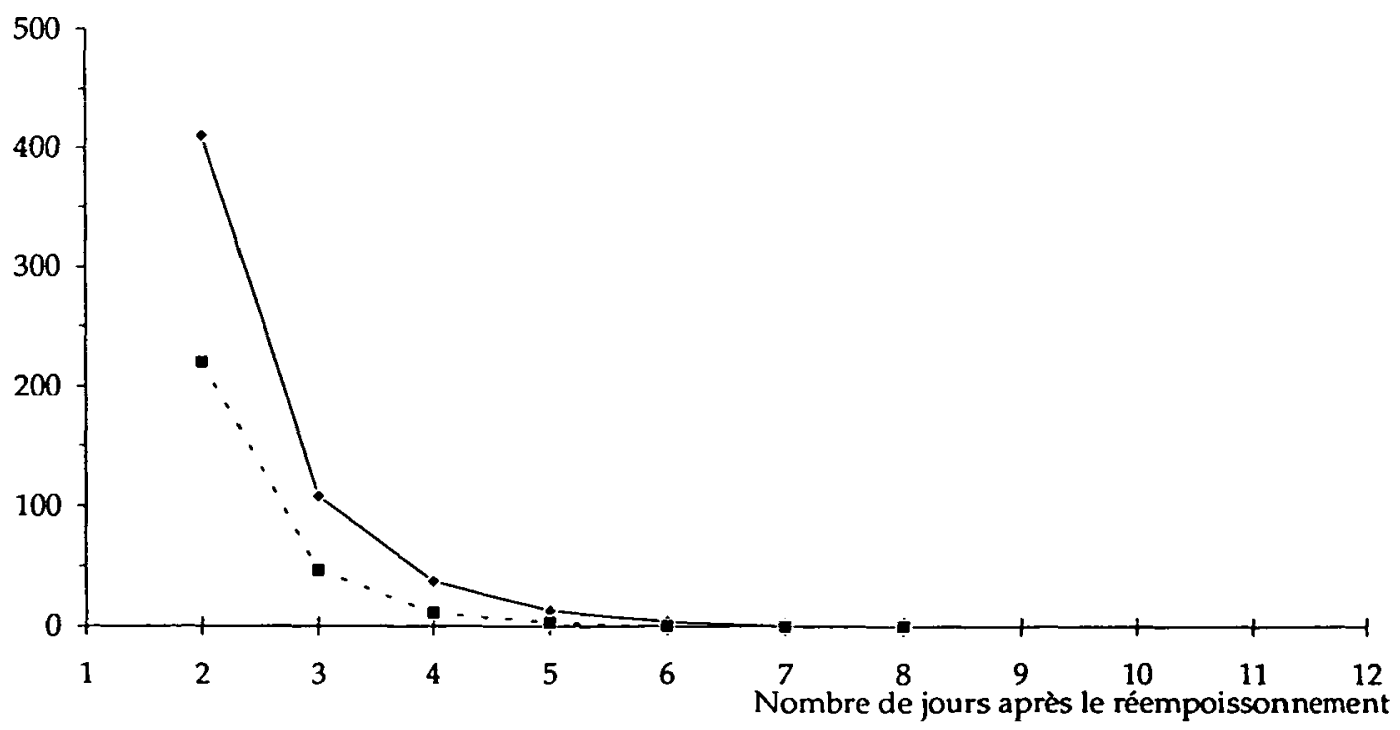

Figure 4 : Répartition temporelle des oiseaux piscivores dans l'enclos non protégé : (a) Héron Cendré, $(\diamond)$ Grand Cormoran.

Figure 4 : Temporal repartition of piscivorous birds in the unprotected enclosure : (E) Grey Heron, $(\bullet)$ Cormorant.

\subsection{Répartition spatiale}

Les cormorans ont exploité le creusement (rectangles 9 à 16), tandis que les hérons se sont cantonnés à la zone peu profonde (rectangles 1 à $8 ; \chi^{2}, p<0,001$, fig. 5). 
La répartition des cormorans dans le creusement n'est pas uniforme $\left(\chi^{2}, p<0,001\right)$, mais elle est aléatoire (test de Kolmogorov-Smirnov, $p>0,05$ ). La répartition des hérons n'est ni uniforme (test de $G$ ajusté, $p<0,001$ ) ni aléatoire (test de Kolmogorov-Smirnov, $p<0,001$ ). Le rectangle $n^{\circ} 1$ le plus proche de la zone protégée est recherché par les hérons, le $n^{\circ} 2$ est neutre, les autres rectangles sont évités (test de contraste au risque de $5 \%$ ). Cette situation n'est pas induite par la position de l'affût susceptible d'effaroucher les oiseaux, les tests restreints aux rectangles les plus éloignés de l'affût ( $n^{\circ} 1$ à 6$)$ donnant les mêmes résultats.

\section{Héron Cendré}

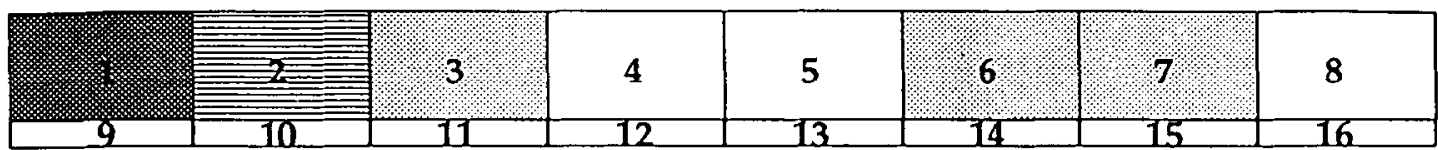

Grand Cormoran

\begin{tabular}{|c|c|c|c|c|c|c|c|}
\hline 1 & 2 & 3 & 4 & 5 & 6 & 1.7 .7 .18 & 8 \\
\hline \hline
\end{tabular}

Fréquence d'observation de l'espèce

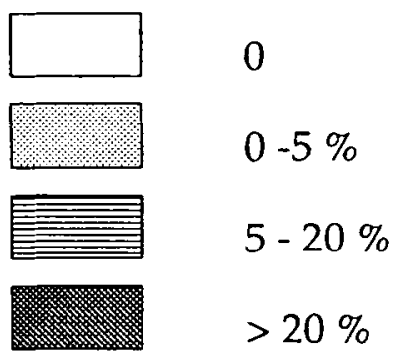

Figure 5 : Répartition spatiale des oiseaux piscivores dans l'enclos non protégé.

Figure 5 : Spatial repartition of piscivorous birds in the unprotected enclosure.

\subsection{Intensité de la pêche}

Le nombre total d'attaques par le héron a été estimé à 50 contre 1200 pour le cormoran, soit 24 fois plus (fig. 6). La comparaison entre les deux espèces des autres composantes du comportement fait apparaître les faits suivants :

- la fréquentation du bassin par les cormorans comparée à celle par les hérons est à peu près deux fois plus importante (respectivement 574 et 305 minutes, fig. 4),

- le temps passé en pêche est comparable (tab. III),

- la durée moyenne d'une pêche est de l'ordre de 4 minutes chez le cormoran et de 7 minutes chez le héron (différence significative, test $t, p<0,025$, tab. III),

- le nombre d'attaques par minute de pêche est supérieur chez le cormoran (2,3 contre $0,1$ chez le héron, $p<0,05)$. 
Tableau III: Quelques caractéristiques de la prédation observées chez le Grand Cormoran et le Héron Cendré (intervalles de confiance à $5 \%$ ).

Table III : Some characteristics of the predation observed among the Cormorant and the Grey Heron.

\begin{tabular}{lcc}
\hline & Cormoran & Héron \\
\hline Temps de pêche $\left(^{*}\right) /$ temps de présence & $70 \%$ & $60 \%$ \\
\hline Durée moyenne d'une pêche $(\mathrm{mn})$ & $4( \pm 1,5)$ & $6,8( \pm 2,2)$ \\
\hline Nombre d'attaques par minute & $2,3( \pm 1,8)$ & $0,1( \pm 0,1)$ \\
\hline
\end{tabular}

( $\left.{ }^{\star}\right)$ estimé sur 260 et 456 minutes de présence respective du Héron Cendré et du Grand Cormoran

Nombre d'attaques

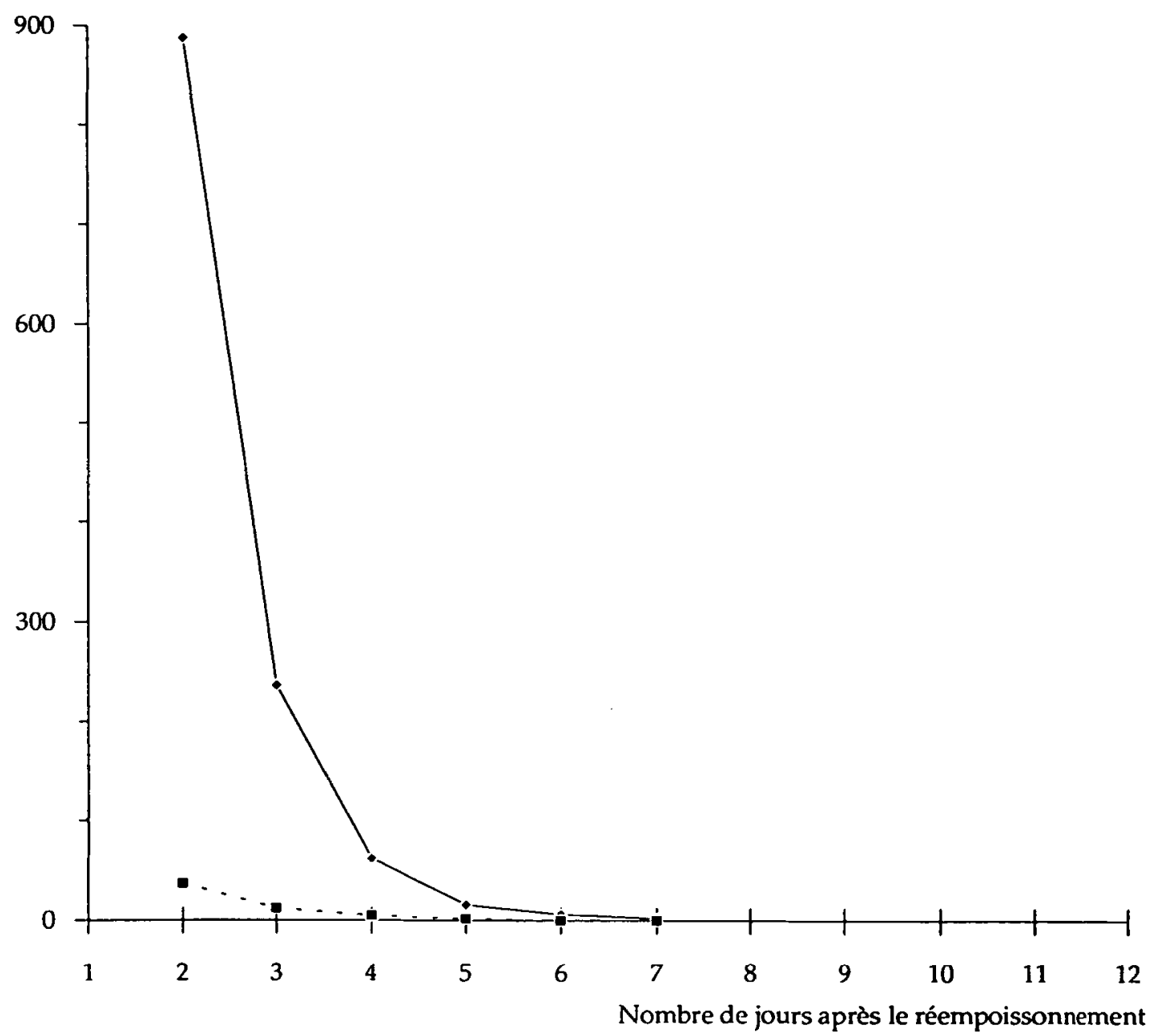

Figure 6 : Nombre d'attaques après empoissonnement. : $(\square)$ Héron Cendré, $(\bullet)$ Grand Cormoran.

Figure 6 : Number of attacks after fish stocking : $(\square)$ Grey Heron, $(\bullet)$ Cormorant. 
La différence observée dans le nombre total d'attaques devrait donc être, en premier lieu, attribuée au nombre d'attaques par minute de pêche et, à un moindre degré, à la fréquentation du bassin. Cette distinction est cependant imprécise car la signification de certaines composantes varie dans le temps. Ainsi, chez le cormoran, la durée moyenne de chaque attaque (plongée) diminue significativement entre le début et la fin de l'expérimentation (de 10 à 4 secondes en moyenne, test de Jonckheere, $p<0,001$, cf. fig. 7). II est possible que les cormorans profitent de densités de peuplement élevées en début de période pour capturer plusieurs proies en une seule plongée, la durée de la plongée étant alors un peu plus importante.

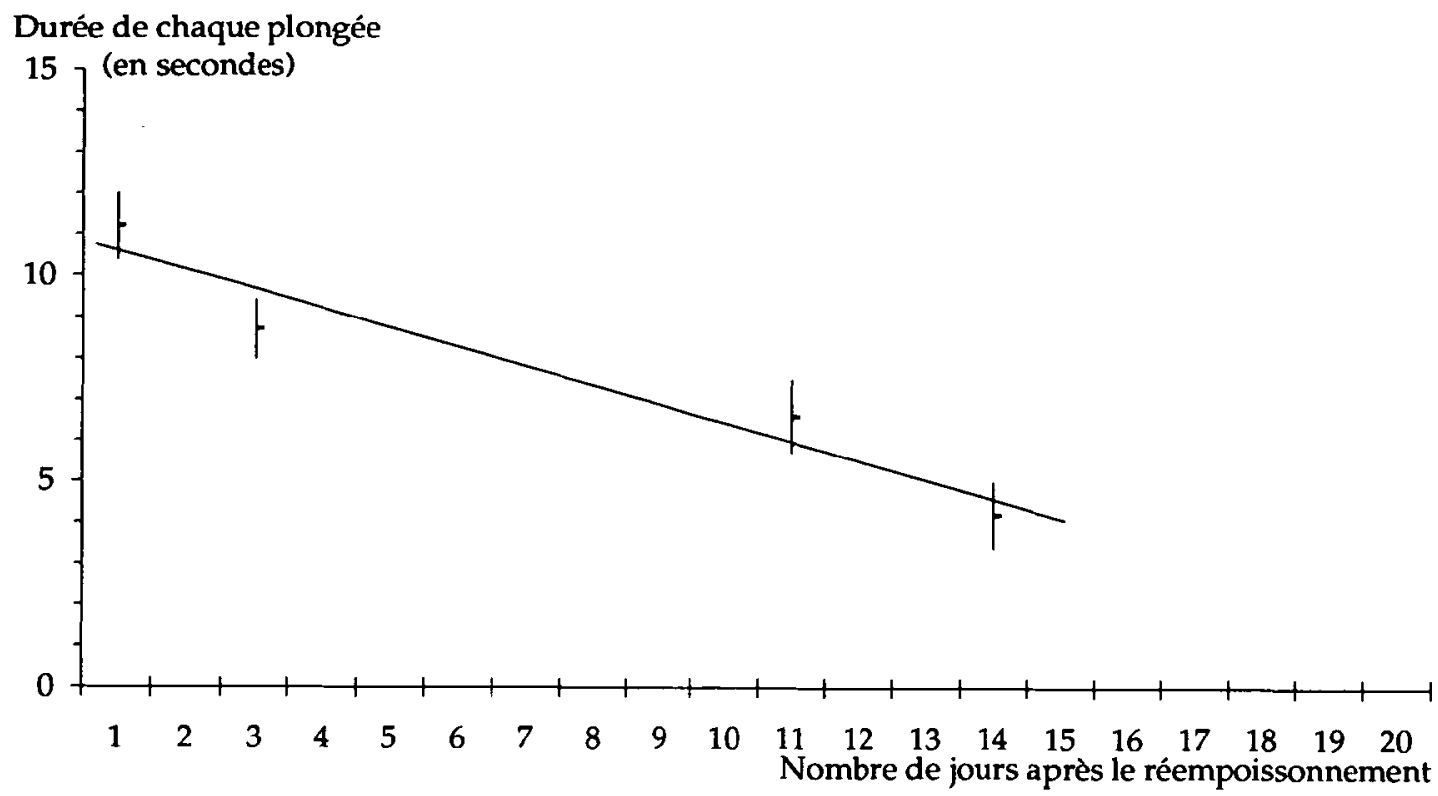

\section{Figure 7 : Durée moyenne de chaque plongée du Grand Cormoran (secondes) en fonction du temps écoulé depuis l'empoissonnement.}

Figure 7 : Mean duration of diving among Cormorants as a function of time elapsed since fish stocking.

\subsection{Impact sur la population de poissons}

La pêche finale de l'enclos soumis à prédation a donné un résultat nul. La disparition complète des poissons n'a ainsi permis de préciser ni le rythme de décroissance du stock, ni un éventuel effet de la taille des poissons. Si l'on se réfère à l'évolution de la fréquentation du bassin par les oiseaux, il est hautement probable que dès le sixième jour la quasi-totalité du stock de poissons avait disparu.

Les résultats de la pêche en zone protégée ont été utilisés pour estimer globalement la mortalité naturelle. Le taux de mortalité naturelle ainsi obtenu $(32,7 \% \pm 2,2 \%$ pour les deux lots confondus) appliqué au peuplement de l'enclos soumis à prédation donne un nombre total de morts de $362 \pm 24$ poissons.

L'estimation de la quantité consommée par le héron est de 33 poissons, soit $3 \%$ du stock initial. Le taux de succès pour cette espèce (nombre de prises / nombre d'attaques) est d'environ $70 \%$.

La prédation par le cormoran n'a pu être quantifiée par comptage direct. On peut calculer, en appliquant le taux de mortalité naturelle du témoin, l'ensemble des pertes dues à la prédation par le cormoran et aux effets indirects des prédateurs (stress, blessures). Si on applique ce taux avant que la prédation n'intervienne, le calcul évalue les pertes par prédation à 711 
poissons, soit $64 \%$ du stock initial. La réalité se situe certainement entre ce nombre et le nombre de poissons non consommés par les hérons, soit 1073 poissons (cas où tous les poissons sont victimes de la prédation avant la mort naturelle). A ces deux hypothèses extrêmes, correspondraient pour le cormoran des taux de succès lors des attaques de 60 à $90 \%$.

\section{DISCUSSION}

Cormorans et hérons sont arrivés sur la zone expérimentale aussitôt l'empoissonnement réalisé. Ceci reflète d'une part la situation particulière du domaine de Certes, zone protégée utilisée comme reposoir par une importante population de hérons et de cormorans en hivernage, d'autre part la capacité qu'ont ces oiseaux à localiser rapidement de nouvelles sources de nourriture. Le peuplement ne présentait pas quantitativement de caractéristiques particulières, mais il parait certain que, malgré les précautions prises, le transfert des poissons, en produisant un regroupement de proies faciles, a contribué à attirer les prédateurs. II faut noter que ce phénomène d'attrait est très probablement un facteur essentiel de la prédation sur l'ensemble du domaine, car il se manifeste régulièrement lorsque des concentrations de poissons se forment soit journellement au pied des écluses à marée montante, soit à la suite de manoeuvres particulières par l'exploitant (renouvellement d'eau, mise à sec des plats, pêche commerciale).

La différence de comportement entre les deux espèces selon la profondeur du bassin est liée à des modes de chasse particuliers. La concentration des hérons à proximité de l'enclos protégé peut s'expliquer soit par un attrait visuel par les poissons de cet enclos, soit plutôt, puisqu'il y avait captures, par une concentration de poissons dans cette zone. Réfugiés dans la partie plus profonde soit pour y trouver un milieu plus tempéré, soit par crainte des prédateurs, ils cherchent à fuir les cormorans et à l'extrémité de l'enclos n'ont d'autre ressource que de longer la clôture vers les parties moins profondes. De telles associations entre cormorans et Ardéidés ont été notées par divers auteurs (FRASER, 1974, IM et HAFNER, 1984, MOERBEEK et al., 1987).

L'impact direct du héron sur la population de poissons a été faible. Ceci correspond aux observations faites par HAFNER et MOSER (1980), GEIGER (1984), UTSCHICK (1984 a et b), PERENNOU (1987) et MARION et MARION (1987). L'estimation de l'impact du cormoran est plus délicate. On ne peut distinguer entre prédation directe et mortalité indirecte due à l'ensemble de la prédation. Selon que l'on attribue ou non l'ensemble de cette mortalité au cormoran, on calcule un taux de succès compris entre 60 et $90 \%$, ce qui est élevé par rapport à celui indiqué par IM et HAFNER (1984) en Camargue (22\%). L'hypothèse d'un taux de succès aussi bas appliqué à notre essai signifierait que la mortalité indirectement due aux prédateurs est très élevée, ou que les plongées des premiers jours permettent des captures multiples. De toute manière, cette comparaison n'est qu'indicative car ce taux dépend étroitement des conditions locales (présence d'abris, conformation du bassin, transparence de l'eau) et des espèces concernées (capacité de fuite, comportement collectif), et il est probable que, dans notre essai, la majeure partie de la mortalité résulte de la prédation directe par le cormoran. L'importance des dégâts causés par cette espèce a déjà été soulignée par plusieurs auteurs (BARLOW et BOCK, 1984 ; IM et HAFNER, op. cit. ; MOERBEEK et al., 1987).

\section{CONCLUSION}

Les résultats obtenus en situation expérimentale sur le domaine de Certes montrent que le Grand Cormoran peut réduire de façon importante un stock de poissons introduit dans un bassin de taille importante $(0,9$ ha). Par contre, le Héron Cendré n'a effectué qu'un faible prélèvement, malgré des conditions favorables (faible profondeur sur une grande partie de la surface, longueur relative des rives importante, action de pêche facilitée par le rabattage par les cormorans).

Des efforts importants ont été faits pour réaliser cette étude dans des conditions comparables à celles rencontrées dans le cadre de la pisciculture extensive telle qu'elle est pratiquée dans les réservoirs du domaine. Parmi les biais induits par l'approche expérimentale, les plus importants paraissent être le choc lié au transfert des poissons, qui facilite certainement le repérage par les prédateurs, et l'uniformité du bassin dépourvu d'abris naturels et de refuges. Mais ces conditions peu naturelles sont fréquemment réunies dans le cadre de la gestion piscicole qui, pour faciliter l'exploitation et améliorer les rendements, uniformise les bassins 
et favorise les regroupements de poissons de façon volontaire ou fortuite (utilisation de bassins d'hivernage, concentration du cheptel pour les pêches commerciales, manoeuvres d'écluses créant des mouvements d'eau attirant les poissons). Ces pratiques favorisent directement la prédation et accroissent les risques par rapportà ceux encourus par les populations naturelles. Ces risques constituent un enjeu capital pour les exploitations à caractère extensif, et des solutions doivent être cherchées en élaborant entre autres des modes de gestion piscicole adaptés à la présence des prédateurs ; la protection totale est en effet efficace à petite échelle, mais d'une application tout à fait inconcevable sur de vastes surfaces. Les très faibles résultats de production enregistrés depuis 1988 sur ce domaine, malgré l'effort particulier porté àl'alevinage naturel et l'absence d'hiver très rigoureux, paraissent confirmer l'impact considérable de la prédation exercée par les oiseaux piscivores constaté sur ce bassin expérimental.

\section{REMERCIEMENTS}

Nous remercions le Conservatoire de l'Espace Littoral et des Rivages Lacustres qui a permis la réalisation de ce travail sur le domaine de Certes. Cette étude a été financée par le SRETIE (Ministère de l'Environnement) et par le contrat Etat-Région Aquitaine "Recherche et développement en aquaculture extensive". Nous remercions également les membres du CROAP (Centre Régional Ornithologique Aquitaine Pyrénées) et A. CALLEC qui nous ont communiqué les résultats de leurs comptages.

\section{BIBLIOGRAPHIE}

ANONYME, 1988. Programme de recherche et de développement en aquaculture extensive. Compte-rendu d'activité 1986 et 1987. CEMAGREF-IUBM Arcachon/AGEDRA, $88+38 \mathrm{p}$.

BARLOW C.G., BOCK K., 1984. Predation of fish in farm dams by cormorants Phalacrocorax spp. Aust. Wildl. Res., 11, 559-566.

BUILLES A., JULLIEN J.M., YESOU P., GIRARD O., 1986. Rythme d'activite et occupation de l'espace par le Grand Cormoran (Phalacrocorax carbo) sur un site d'hivernage : l'exemple de la région d'Olonne-Vendée. Gibier Faune sauvage, 3, 43-65.

BOWMAKER A.P., 1963. Cormorant predation on two Central Africa lakes. Ostrich, 31, 1, 2-26.

DOORNBOS G., 1984. Piscivorous birds on the saline lake Grevelingen, Netherlands : abundance, prey selection and annual food consumption. Netherlands Journal of Sea Research, 18, 457-479.

EIFAC, 1988. Report of the EIFAC Working Party on prevention and control of bird predation in aquaculture and fisheries operations. EIFAC, Tech. Pap., 51, $79 \mathrm{p}$.

FRASER W., 1974. Feeding association between Little Egret and Reed Cormorant. Ostrich, $45,262$.

GEIGER C., 1984. Graureiher Ardea cinerea und Fischbestand in Fliessgewässern. Der Ornithologische Beobachter, 81, 111-131.

GERDEAUX D., 1987. Revue des méthodes d'estimation de l'effectif d'une population par pêches successives avec retrait. Programme d'estimation d'effectifs par la méthode de Carle et Strub. Bull. Fr. Pêche Piscic., 304, 13-21.

HAFNER H., MOSER M., 1980. Les hérons et la pisciculture en Camargue. Bull. ONC Scient. Tech., 255-260.

IM B.H., HAFNER H., 1984. Impact des oiseaux piscivores et plus particulièrement du Grand Cormoran (Phalacrocorax carbo sinensis) sur les exploitations piscicoles en Camargue. Contrat de recherche CEE ENV.491-F, Station biologique de La Tour du Valat, $84 \mathrm{p}$.

IM B.H., HAFNER H., 1985. Impact des oiseaux piscivores et plus particulièrement du Grand Cormoran (Phalacrocorax carbo sinensis) sur les exploitations piscicoles en Camargue. Bull. ONC, 94, 30-36. 
LABOURG P.J., 1976. Les réservoirs à poissons du Bassin d'Arcachon et l'élevage extensif de poissons euryhalins (muges, anguilles, bars et daurades). Pisc. Franç., 45, 35-52.

MARION L., 1983. Rapport du Groupe de Travail sur les Oiseaux Piscivores.

MARION L., MARION P., 1987. Impact de la prédation exercée par le Héron Cendré sur une pisciculture. Rev. Ecol. Terre \& Vie, suppl. 4, 115-116.

MOERBEEK D.J., VAN DOBBEN W.H., OSIECK E.R., BOERE G.L., BUNGENBERG DE JONG C.M., 1987. Cormorant damage prevention at a fish farm in the Netherlands. Biol. Conserv., 39, 27-38.

PARKHURST J.A., BROOKS R.P., ARNOLD D.E., 1987. A survey of wildlife depredation and control techniques at fish-rearing facilities. Wildl. Soc. Bull., 15, 386-394.

PERENNOU C., 1987. L'impact du Héron Cendré Ardea cinerea en bassin d'alevinage. L'oiseau et R.F.O., 57, 262-265.

PINOWSKI J., MORALES L.G., PACHECS J., DOBROLOWSKI K.A., PINOWSKA B., 1980. Estimation of the food consumption of fish-eating birds in the seasonally-flooded savanas (llanos) of Alto Apure, Venezuela. Bull. Acad. Polonaise Sci., serie Sci. Biol.,28, 163-170.

SCHERRER B., 1984. Biostatistique. Gaëtan Morin, Chicoutimi, 850 p.

UTSCHICK V. H., 1984 a. Ökologische Untersuchungen zur Rolle des Graureihers Ardea cinerea in der Sportfischerei. Verh. Orn. Ges. Bayern, 24, 87-110.

UTSCHICK V. H., 1984 b. Untersuchungen zur Rolle des Graureihers Ardea cinerea in der Teichwirtschaft. Verh. Orn. Ges. Bayern, 24, 111-124. 